\title{
Long-Range Correlations in the Wave Functions of Chaotic Systems
}

\author{
Vladimir I. Fal'ko ${ }^{1,3,4}$ and K. B. Efetov ${ }^{2,5}$ \\ ${ }^{1}$ School of Physics \& Chemistry, Lancaster University, LA1 4YB Lancaster, United Kingdom \\ ${ }^{2}$ Max-Planck-Institut für Physik Komplexer Systeme, Heisenbergstrasse 1, 70569 Stuttgart, Germany \\ ${ }^{3}$ Department of Theoretical Physics, Oxford University, 1 Keble Road, OX1 3NP, Oxford, United Kingdom \\ ${ }^{4}$ Institute of Solid State Physics RAS, Chernogolovka, 142432 Russia \\ ${ }^{5}$ L. D. Landau Institute for Theoretical Physics, Kosygina 1, Moscow, Russia
}

(Received 12 February 1996)

We study correlations of the amplitudes of wave functions of a chaotic system at large distances. For this purpose, a joint distribution function of the amplitudes at two distant points in a sample is calculated analytically using the supersymmetry technique. The result shows that although in the limit of the orthogonal and unitary symmetry classes the correlations vanish, they are finite through the entire crossover regime and may be reduced only by localization effects. [S0031-9007(96)00681-3]

PACS numbers: 73.20.Dx, 05.45.+b, 71.20.-b

During the past decade, a substantial progress has been achieved in the studies of quantum properties of classically chaotic systems [1]. At present, we have quite a complete information about energy spectra of nonintegrable systems in the limit of a "hard" chaos as well as in the crossover regimes to integrability or localization. However, the theoretical background for understanding the statistics and structure of wave functions of chaotic systems is still a growing-up field stimulated by the recent experiments on electron transport in quantum dots under the Coulomb blockade conditions [2] and on the microwave irradiation in disordered and chaotic cavities [3]. According to the resonant tunneling models of the transport in the Coulomb blockade devices [4,5], the height $g$ of the conductance peaks measured in the experiments on the quantum dots can be related to the local amplitudes $\varphi_{\alpha}\left(\mathbf{r}_{l, r}\right)$ of the wave function of the resonant level $\epsilon_{\alpha}$ as

$$
g=\frac{e^{2} / h}{4 \pi T} \frac{\Gamma_{l} \Gamma_{r}}{\Gamma_{l}+\Gamma_{r}},
$$

where broadening $\Gamma_{l, r} \propto\left|\varphi_{\alpha}\left(\mathbf{r}_{l, r}\right)\right|^{2}$ is due to the electron escape to the right- and left-hand-side electrodes [6]. Hence the statistics of the resonant tunneling conductances in this kind of measurements is determined by the statistics of the electron wave functions in the dot. Moreover, the statistics and spatial correlation properties of microwaves in random media have become the subject of direct measurements performed already in various parametric regimes [3].

Below, we demonstrate the existence of long-range correlations in the wave functions of a chaotic system subjected to a weak magnetic field, that is, in the crossover regime between the orthogonal (with time-reversal symmetry) and unitary (broken time-reversal symmetry) ensembles. This conclusion is based on the analysis of the joint probability distribution function $f_{2}\left(p_{1}, p_{2}\right)$, which describes the statistics of local densities of an eigenstate in a chaotic cavity taken at two distant points in the sample and its comparison with the statistics of a single- point distribution $f_{1}\left(p_{1}\right)$. The joint distribution functions $f_{N}\left(p_{1}, \ldots, p_{N}\right)$ of local densities of a wave function are defined for an arbitrary $N$ as follows:

$$
f_{N}=\Delta\left\langle\sum_{\alpha} \delta\left(\epsilon-\epsilon_{\alpha}\right) \prod_{n=1}^{N} \delta\left(p_{n}-\left|\varphi_{\alpha}\left(\mathbf{r}_{n}\right)\right|^{2}\right)\right\rangle,
$$

where the angular brackets stand for averaging over irregularities in the system or over energies $\epsilon$ [7] in a chaotic billiard, $V$ is the volume of the system, and $\Delta$ is the mean level spacing. The sum in Eq. (1) is taken over all eigenstates, $\varphi_{\alpha}$ and $\epsilon_{\alpha}$ being eigenfunctions and eigenenergies. Because of the normalization of the distribution function $f_{N}$ and wave functions themselves, the lower order functions $f_{N-1}$ can be related to the higher order ones after integrating over some of variables [8]. The joint probability distribution function $f_{2}$ naturally appears in the description of fluctuations of the resonant tunneling conductance peaks, $g$, in quantum dots or can be directly studied in the microwave experiments [3].

Direct computation of the local density distribution function $f_{1}$ for disordered and chaotic systems in the limiting cases of the orthogonal and unitary ensembles $[5,9,10]$ leads to the well-known Porter-Thomas formula [11], which is merely a Gaussian distribution of wave function amplitudes. The Gaussian distribution has been recently confirmed by the numerical studies of high-lying chaotic eigenstates [12]. It can also be derived semiclassically assuming that classical orbits cover uniformly the energy surface in the phase space [13]. The detailed analysis of the joint distribution function $f_{2}$ performed in Ref. [14] for these two universality classes has shown that intrinsic correlations in the wave functions of a chaotic system decay already on several wavelengths, and the function $f_{2}$ can be written at large distances as

$$
f_{2}\left(p_{1}, p_{2}\right) \longrightarrow f_{1}\left(p_{1}\right) f_{1}\left(p_{2}\right),
$$

where the function $f_{1}(p)$ describes the Gaussian distribution of local amplitudes $\varphi\left(\mathbf{r}_{n}\right), p_{n} \equiv V\left|\varphi\left(\mathbf{r}_{n}\right)\right|^{2}$. Moreover, one can provide a full analytical description of a 
decay of correlations of local amplitudes of chaotic wave functions in each of the symmetry classes $[14,15]$. The law describing the decay of the correlations can be derived either using the supersymmetric $\sigma$-model technique or with Berry's conjecture [13] about the uniform distribution of the classical orbits in the phase space [15], and the result for the orthogonal case is in a good agreement with that of the microwave experiments [3].

The suppression of correlations within a chaotic wave function at large distances [Eq. (2)] seems to be so natural for systems in which the semiclassical version of quantum mechanics deals with large random phases that it was not called into question also in the regime of a crossover between the orthogonal and unitary symmetry classes. In this Letter we show that due to the Aharonov-Bohm effect, the intrinsic correlations implicit in a chaotic wave function do not vanish at large distances in the entire crossover regime, although they do in the orthogonal and unitary limits (corresponding to zero and sufficiently high magnetic fields, respectively). In weak magnetic fields, the correlations survive at distances $r=\left|\mathbf{r}_{1}-\mathbf{r}_{2}\right|$ much longer than the mean free path $l$ [that is, $f_{2}\left(p_{1}, p_{2}\right) \neq$ $\left.f_{1}\left(p_{1}\right) f_{1}\left(p_{2}\right)\right]$.

Calculations presented below are performed using the supersymmetry technique [16]. In particular, the use of this method enables one to calculate the one-point distribution function $f_{1}(p)$ for an arbitrary magnetic field [10] (a somewhat different although related quantity was calculated in Ref. [17]). The function $f_{2}$ can be computed in a similar way. Although the calculations are not more difficult than those in Ref. [10], they lead to the result about the long-range correlations which could not be extracted from the function $f_{1}$ alone.

To carry out the computations within the supersymmetry scheme, one should write the quantities to be studied in terms of Green functions defined, as usual, as

$$
G_{\epsilon}^{R, A}\left(\mathbf{r}_{1}, \mathbf{r}_{2}\right)=\sum_{\alpha} \varphi_{\alpha}\left(\mathbf{r}_{1}\right) \varphi_{\alpha}^{*}\left(\mathbf{r}_{2}\right) /\left[\epsilon-\epsilon_{\alpha} \pm i \gamma / 2\right]
$$

It is not difficult to show that the distribution function $f_{2}$, Eq. (1), can be represented as follows:

$$
f_{2}\left(p_{1}, p_{2}\right)=\frac{\Delta}{\pi} \lim _{\substack{\beta \rightarrow 1 \\ \gamma \rightarrow 0}} \frac{\partial}{\partial \beta}\left\langle\beta \int_{0}^{1} d t \int d \mathbf{r} \operatorname{Im}\left[G_{\epsilon}^{A}(\mathbf{r}, \mathbf{r})\right] \delta\left[p_{1}+i Y_{A} G_{\epsilon}^{A}\left(\mathbf{r}_{1}, \mathbf{r}_{1}\right)\right] \delta\left[p_{2}-i Y_{R} G_{\epsilon}^{R}\left(\mathbf{r}_{2}, \mathbf{r}_{2}\right)\right]\right\rangle,
$$

where $Y_{A}=\gamma \beta V t$ and $Y_{R}=\gamma \beta V(1-t)$. Next, we express the Green functions entering Eq. (3) in terms of integrals over supervectors $\psi$ with the Lagrangian $L$,

$$
L[\psi]=i \int \bar{\psi}(\mathbf{r})\left[\epsilon-\hat{H}_{0}-U(\mathbf{r})-i \gamma \Lambda / 2\right] \psi(\mathbf{r}) d \mathbf{r},
$$

where $\hat{H}_{0}$ is the free particle Hamiltonian and $U(\mathbf{r})$ describes the impurity potential. The diagonal matrix $\Lambda$ has components $\Lambda^{11}=-\Lambda^{22}=1$, and $\psi$ is an eightcomponent supervector containing both commuting $s$ and anticommuting $\chi$ elements. The reduction of Eq. (3) to the integral over the supervectors $\psi$ can be done by expanding $\delta$ functions in the Green functions $G_{\epsilon}^{R}$ and $G_{\epsilon}^{A}$ and using standard formulas of Gaussian integration. Then one averages over the irregularities and, finally, uses the Hubbard-Stratonovich transformation to decouple the "interaction" term $(\bar{\psi} \psi)^{2}$ obtained after this averaging by an integration over the supermatrix field $Q$. This gives us a possibility to integrate over the supervectors $\psi$ and to reduce the following computation to that of an integral over the supermatrices $Q$. Integrating over the supervector $\psi$, we take into account only pairing at coinciding points. The approximation is justified provided the distance between the points $\mathbf{r}_{1}$ and $\mathbf{r}_{2}$ is large enough (much larger than the wavelength). This means that we consider the asymptotic form of the distribution function of local amplitudes of the wave function taken at essentially distant points. All manipulations are standard $[10,16]$, and we get

$$
f_{2}\left(p_{1}, p_{2}\right)=\lim _{\substack{\beta \rightarrow 1 \\ \gamma \rightarrow 0}} \frac{d}{d \beta}\left\langle\frac{\beta}{2} \int \frac{d \zeta_{1} d \zeta_{2}}{(2 \pi)^{2}} \int_{0}^{1} d t\left(Q_{11}^{11}-Q_{11}^{22}\right) \delta\left(p_{1}-\frac{t \gamma \beta}{2 \Delta} \bar{z}_{1} Q z_{1}\right) \delta\left(p_{2}+\frac{(1-t) \gamma \beta}{2 \Delta} \bar{z}_{2} Q z_{2}\right)\right\rangle_{Q},
$$

where $\langle\cdots\rangle_{Q}$ stands for the integration over supermatrices $Q$ with the free energy $F[Q]$, and $\bar{z}_{2}=\left(0,0,0,0,0,0, e^{i \zeta_{2}}, e^{-i \zeta_{2}}\right), \bar{z}_{1}=\left(0,0, e^{i \zeta_{1}}, 0,0,0,0\right)$. In the presence of a magnetic field, $F[Q]$ is given by [18]

$$
\begin{aligned}
F & =-\operatorname{Str}\left(\frac{\pi \gamma}{4 \Delta} \Lambda Q+\left(\frac{X}{4}\right)^{2}\left[Q, \tau_{3}\right]^{2}\right), \\
X^{2} & =2 \pi \frac{D}{\Delta} \int \frac{d \mathbf{r}}{V}\left(\frac{2 \pi \mathbf{A}(\mathbf{r})}{\phi_{0}}\right)^{2}=\alpha_{g} \frac{\phi^{2}}{\phi_{0}^{2}} \frac{E_{c}}{\Delta},
\end{aligned}
$$

where $\phi_{0}=h c / e$ is the flux quantum, $\alpha_{g}$ is the sample geometry dependent factor, $D$ is the classical diffusion coefficient, and $E_{c}$ is the Thouless energy. Equations (4) and (5) are written under the assumption that the supermatrix $\sigma$ model describing fluctuations of the supermatrix $Q$ is zero dimensional (0D), which is correct for not very large $p_{1,2}$. The distribution function $f_{2}$ can also be calculated for arbitrary $p_{1,2}$ in a way that has been done for the function $f_{1}$ in Ref. [19]. This would require going 
beyond the 0D $\sigma$ model and is out of the scope of this publication.

Calculation of the integral over $Q$ in Eq. (4) can be carried out using the parametrization suggested in Ref. [18], which separates "Cooperon" and "diffuson" variables. The quantum limit $\gamma \rightarrow 0$ considerably simplifies the integration over the diffuson variables, since the main contribution comes from that part of the noncompact sector of the order parameter $Q$ where $\left|\bar{z}_{n} Q z_{n}\right| \sim$ $1 / \gamma \rightarrow \infty$. In this limit, the diffuson degrees of freedom can be integrated out, and one ends up with a version of some kind of a reduced $\sigma$ model adapted for describing the properties of a single quantum state [19] and containing only the integration over the Cooperon variables,

$$
\begin{aligned}
f_{N}\left(p_{1}, \ldots, p_{N}\right)= & \int_{0}^{1} d \lambda_{c} \int_{1}^{\infty} d \lambda_{1 c} \exp \left(-F_{\phi}\right) \\
& \times \frac{\lambda_{c}^{2}}{\left(\lambda_{1 c}^{2}-\lambda_{c}^{2}\right)^{2}} \int P \prod_{n=1}^{N} \frac{d \zeta_{n}}{2 \pi} \\
& \times \frac{\exp \left(-p_{n} / A_{n}\right)}{A_{n}} d R_{c} .
\end{aligned}
$$

Hereby, $N=1,2, A_{n}=1+\operatorname{Re}\left(L e^{i \zeta_{n}} / P\right)$ and

$$
P=\lambda_{1 c}+2\left(\eta_{c} \eta_{c}^{*}-\kappa_{c} \kappa_{c}^{*}\right)\left(\lambda_{1 c}-\lambda_{c}\right), \quad L=\sqrt{\lambda_{1 c}^{2}-1}\left(1+2 \eta_{c} \eta_{c}^{*}\right)\left(1-2 \kappa_{c} \kappa_{c}^{*}\right)+4 \sqrt{1-\lambda_{c}^{2}} \eta_{c} \kappa_{c} .
$$

Note that $d R_{c}=d \eta_{c} d \eta_{c}^{*} d \kappa_{c}^{*} d \kappa_{c}$, with $\eta_{c}, \eta_{c}^{*}, \kappa_{c}, \kappa_{c}^{*}$ being Grassmann anticommuting variables.

Skipping technical details of the algebraic manipulations, we are able to represent the joint distribution function $f_{N}, N=1,2$, in the form

$$
f_{N}=\int_{1}^{\infty} B(X, x) \prod_{n=1}^{N} M\left(p_{n}, x\right) d x,
$$

where

$$
\begin{aligned}
B(X, x) & =X^{2}\left[\left(x X^{2}-1\right) \Phi_{2}(X)+\Phi_{1}(X)\right] e^{-X^{2}(x-1)}, \\
M(p, x) & =\sqrt{x} \exp (-p x) I_{0}\left(p \sqrt{x^{2}-x}\right) .
\end{aligned}
$$

Here $I_{0}(z)$ is the modified Bessel function, and

$$
\Phi_{1}(X)=\frac{e^{-X^{2}}}{X} \int_{0}^{X} e^{y^{2}} d y, \quad \Phi_{2}(X)=\frac{1-\Phi_{1}(X)}{X^{2}} .
$$

The joint distribution function of this form satisfies the normalization condition described in footnote [8].

In order to establish the presence or absence of correlations between fluctuations of the values $p_{1,2} \equiv\left|\varphi\left(\mathbf{r}_{1,2}\right)\right|$ at distant points in the sample in the crossover regime, the joint probability distribution function $f_{2}$ and its moments have to be compared to the distribution function $f_{1}$. Explicit integration over $x$ in Eqs. (6) shows that in each of two limits $X \rightarrow 0$ and $X \rightarrow \infty, f_{2}\left(p_{1}, p_{2}\right)$ takes a separable form and satisfies Eq. (2). That is, $f_{2}\left(p_{1}, p_{2}\right)=\Pi_{i=1,2} e^{-p_{i} / 2} / \sqrt{2 \pi p_{i}}$ in the orthogonal case and $f_{2}\left(p_{1}, p_{2}\right)=\exp \left(-p_{1}-p_{2}\right)$ in the unitary one. However, the distribution function is not separable at any finite magnetic field, which means that even in the limit of a large distance between the points $\mathbf{r}_{1}$ and $\mathbf{r}_{2}$ the correlations do not vanish.

To illustrate the existence of long-range correlation implicit in each individual wave function in a quantum billiard subjected to a weak magnetic field, we calculate the correlation function $K_{2,2}(X)$ defined as [20]

$$
K_{2,2}=\int_{0}^{\infty} d p_{1} d p_{2} p_{1}^{2} p_{2}^{2}\left[f_{2}\left(p_{1}, p_{2}\right)-f_{1}\left(p_{1}\right) f_{1}\left(p_{2}\right)\right] .
$$

Although an analytical expression for $K_{2,2}$ can be found from Eqs. (6) using the identity from footnote [8], we give here only its graphic representation. Figure 1 illustrates how the correlations evolve as a function of a normalized flux through the sample area. As one can see from Fig. 1, the correlations are present all over the crossover regime: from the smallest to highest values of the normalized flux $\phi$. These correlations are never strong (of the order of $1 \%$ ), since the maximal value of the correlator $\max \left(K_{2}\right) \approx$ 0.05 has to be compared to the square of the second moment $P_{2}=\int d p p^{2} f_{1}(p)$, which varies from the value of 3 to 2 in between the orthogonal and unitary symmetry classes.

The distant correlations in the wave functions can be also traced in the joint probability to find simultaneously two zeros of the wave function $\varphi\left(\mathbf{r}_{1,2}\right)=$ 0 . At small fluxes corresponding to $X \ll 1,\left[f_{1}(0)\right]^{2} \approx$ $\frac{4 \pi}{9} X^{-2}$, whereas $f_{2}(0,0) \approx \frac{5}{3} X^{-2}$. Since the crossover between the orthogonal and unitary ensembles occurs at relatively small magnetic fluxes through the sample area, $\phi \sim \phi_{0}\left(\Delta / E_{c}\right)^{1 / 2}$, the nonseparability of $f_{2}\left(p_{1}, p_{2}\right)$ as a whole is most pronounced when $X \sim 1$. In Fig. 2 we show the difference between $f_{2}$ and $f_{1} f_{1}$ at $X=1$.

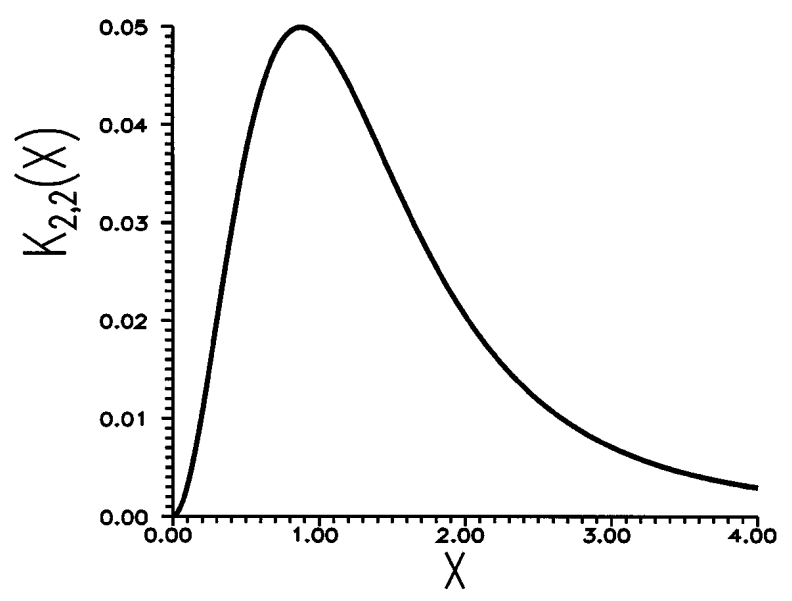

FIG. 1. The correlation function $K_{2,2}(X)$ as a measure of spatial correlation implicit to a chaotic wave function. 


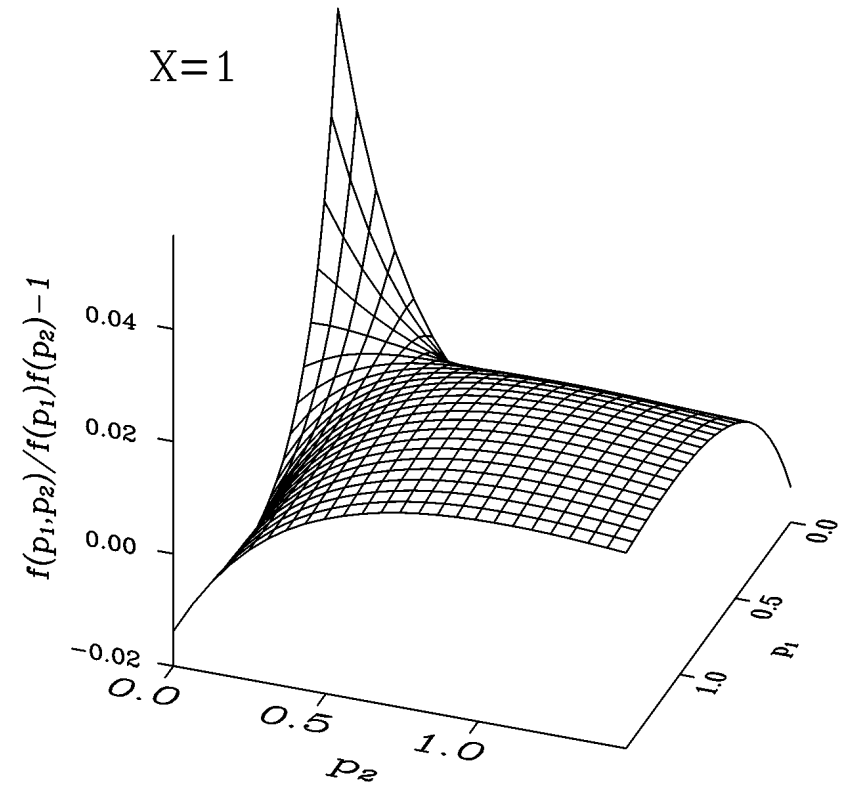

FIG. 2. The difference between $f_{2}$ and $f_{1} f_{1}$ normalized by $f_{1} f_{1}$ for $X=1$.

Starting from the pioneering work by Berry [13], it was generally believed that in classically ergodic systems the local wave function density can be imagined as a result of superposition of an infinite number plane waves with random phases and equal momenta. The randomness of the phases suggests the Gaussian randomness of the amplitude $\varphi_{\alpha}$ and vanishing correlations at large distances. Our result means that a weak magnetic flux through the sample area introduces some correlations in $\varphi_{\alpha}(\mathbf{r})$ related to the fact that the Aharonov-Bohm phases taken by an electron moving in a quantum billiard cannot be arbitrarily large. The lengths of geometrical paths attributed to an electron classical trajectory in a semiclassical picture of quantum mechanics are limited, since the semiclassics breaks down at the time scale longer than the Heisenberg time, $t_{H} \sim h / \Delta$. Hence the encircled magnetic field fluxes are also limited, so that the classical ergodicity does not always lead to the complete randomness of the wave function phases.

The correlations discussed above persist all over the chaotic quantum billiard. One may ask a question about what happens if the sample size is much larger than the mean free path in a disordered system. In fact, the result of Eq. (6) is valid as far as the OD $\sigma$ model may be used, that is, unless localization effects become important. Hence in 1D and 2D weakly disordered samples the distance between the observation points must be smaller than the localization length, whereas in a 3D metal the distance between them is not limited. The correlations can be considerably reduced also for large values of amplitudes originated from prelocalized states. In this case, one has to go beyond the $0 \mathrm{D} \sigma$ model $[19,21]$.
In conclusion, the joint distribution function of amplitudes of chaotic wave functions is derived for an arbitrary magnetic flux. Its form manifests the long-range spatial correlations existing in the entire crossover regime from orthogonal to unitary symmetry classes. This indicates that due to the Aharonov-Bohm effect the phases of the wave functions may be correlated even if the corresponding classical motion is ergodic, which has not been anticipated in previous semiclassical theories.

One of us (V.F.) thanks EPSRC for financial support and MPI-PKS for hospitality.

[1] Chaos and Quantum Systems, edited by M-J. Giannoni, J. Voros, and Zinn-Justin (Amsterdam, North-Holland, 1991); F. Haake, Quantum Signatures of Chaos (Springer, Berlin, 1992).

[2] A. M. Chang et al., Phys. Rev. Lett. 76, 1695 (1996); J. A. Folk et al., ibid. 76, 1711 (1996).

[3] A. Kudrolli, V. Kidambi, and S. Sridhar, Phys. Rev. Lett. 75, 822 (1995); V. N. Prigodin et al., ibid. 75, 2392 (1995).

[4] R. G. Jalabert, A. D. Stone, and Y. Alhassid, Phys. Rev. Lett. 68, 3468 (1992).

[5] V.N. Prigodin, K. B. Efetov, and S. Iida, ibid. 71, 1230 (1993).

[6] This formula for the resonance conductance corresponds to the limit of $T \gg \Gamma$. Other regimes were studied in [5].

[7] O. Agam, B. L. Altshuler, and A. V. Andreev, Phys. Rev. Lett. 75, 4389 (1995); A. V. Andreev et al. (to be published).

[8] The functions $f_{N}\left(p_{1}, \ldots, p_{N}\right)$ obey the normalization conditions $\int_{0}^{\infty} d p_{N} f_{N}\left(p_{1}, \ldots, p_{N}\right)=f_{N-1}\left(p_{1}, \ldots, p_{N-1}\right)$ and $\int_{0}^{\infty} p_{N} d p_{N} f_{N}\left(p_{1}, \ldots, p_{N}\right)=f_{N-1}\left(p_{1}, \ldots, p_{N-1}\right)$. Its validity may be checked in the final result of this paper using the identity $\int_{0}^{\infty} d p \exp (-a p) I_{0}(b p)=\left[a^{2}-\right.$ $\left.b^{2}\right]^{-1 / 2}$.

[9] K. B. Efetov and V. N. Prigodin, Phys. Rev. Lett. 70, 1315 (1993); Mod. Phys. Lett. B 7, 981 (1993); A. D. Mirlin and Y. V. Fyodorov, J. Phys. A 26, L551 (1993).

[10] V. I. Fal'ko and K. B. Efetov, Phys. Rev. B 50, 11267 (1994).

[11] T. A. Brody et al., Rev. Mod. Phys. 53, 385 (1981).

[12] B. Li and M. Robnik, J. Phys. A 27, 5509 (1994).

[13] M. V. Berry, J. Phys. A 10, 2083 (1977).

[14] V.N. Prigodin, Phys. Rev. Lett. 74, 1566 (1995); E. Muciollo et al., ibid. 75, 1360 (1995).

[15] M. Srednicki, Report No. cond-mat/9512115.

[16] K. B. Efetov, Adv. Phys. 32, 53 (1983).

[17] H.-J. Sommers and S. Iida, Phys. Rev. E 49, 2513 (1994); K. Zyczkowsky and G. Lenz, Z. Phys. B 82, 299 (1991).

[18] A. Altland et al., J. Phys. A 26, 3545 (1993); K. B. Efetov and S. Iida, Phys. Rev. B 47, 15794 (1993).

[19] V.I. Fal'ko and K. B. Efetov, Europhys. Lett. 32, 627 (1995); Phys. Rev. B 52, 17413 (1995).

[20] Because of the normalization of the wave function $\varphi_{\alpha}$, $K_{2,2}$ is the lowest-order nontrivial correlation function.

[21] B. A. Muzykantskii and D. E. Khmelnitskii, Phys. Rev. B 51, 5480 (1995). 